[0212-7199 (2008) 25: 5; pp 203-204] ANALES DE MEDICINA INTERNA Copyright (C) 2008 ARAN EDICIONES, S.L.

AN. MED. INTERNA (Madrid) Vol. 25, N. ${ }^{\circ}$ 5, pp. 203-204, 2008

\title{
Nuevos fármacos en el cáncer de mama
}

Khosravi Shahi P, Pérez. Manga G. Nuevos fármacos en el cáncer de mama. An Med Interna (Madrid) 2008; 25: $203-204$.

El cáncer de mama invasivo (CMI) es la neoplasia más frecuente en la mujer en los países occidentales. En las últimas décadas ha habido una disminución de la mortalidad asociada al cáncer de mama (1), debido a las mejoras en su diagnóstico y tratamiento $(2,3)$.

Sin embargo, el CMI no es una entidad clinicopatológica uniforme, sino un grupo heterogéneo de neoplasias originadas en la mama, que presentan una historia natural diferente. El empleo de la tecnología de microarrays del ADN (análisis sincrónico de miles de genes expresados por un mismo tumor) ponen de manifiesto la existencia de al menos tres grandes subtipos genéticos de CMI, con implicación terapéutica y pronostica (4):

1. El subtipo luminal (A, B, y C) se caracteriza por una alta expresión de receptores estrogénicos (RE) y de genes regulados por los estrógenos. Suele asociarse con una alta tasa de respuesta a la hormonoterapia.

2. El subtipo HER-2 positivo se caracteriza por la ausencia de expresión de RE, y una amplificación y/o sobreexpresión del gen HER-2, lo que implica mayor agresividad biológica, y peor pronóstico. Sin embargo, su historia natural ha sufrido un cambio con la introducción en el mercado de nuevos agentes terapéuticos anti-HER-2.

3. El subtipo basal-like se caracteriza por la ausencia de expresión de los receptores estrogénicos, progesterónicos, y HER-2 (fenotipo triple negativo). Sin embargo, no existe una exacta correlación entre el fenotipo triple negativo por inmunohistoquímica y el perfil genético basal-like (5). En un 50\% de los casos presentan una sobreexpresión del receptor de factor de crecimiento epidérmico (EGFR), y generalmente se asocian con hallazgos histopatológicos de alto grado de malignidad (grado nuclear elevado, pleomorfismo o un alto índice mitótico). Por tanto, se trata de un subtipo de CMI de mal pronóstico, pero que de forma paradójica se asocia con una alta tasa de respuesta a la quimioterapia, en especial a las sales de platino, y probablemente a las terapias anti-EGFR.

El tratamiento de CMI es complejo, y habitualmente de carácter multidisciplinar. La quimioterapia basada en fármacos con una acción citotóxica directa sigue siendo parte fundamental del tratamiento de CMI, tanto en los estadios inicia- les, como en la fase metastática. En este sentido se han desarrollado nuevos fármacos citostáticos eficaces en el tratamiento de CMI avanzado.

En primer lugar, cabe mencionar una nueva familia de citostáticos denominados "epotilonas". En esta familia destaca ixabepilona, que ha demostrado su eficacia en un ensayo fase III en la enfermedad metastática tras progresión a taxanos y antraciclinas. En el estudio 752 pacientes fueron asignadas de forma aleatoria a recibir ixabepilona más capecitabina o capecitabina en monoterapia. Se demostró un incremento significativo de la supervivencia libre de progresión (SLP) en la rama de ixabepilona $(5,8$ vs. 4,2 meses; $\mathrm{HR}=0,75 ; \mathrm{p}=$ $0,0003)$, así como un aumento en las respuestas objetivas ( 35 vs. $14 \% ; \mathrm{p}<0,0001)$. La tasa de muerte tóxica fue mayor en la rama de ixabepilona ( $3 v s .1 \%$ ), siendo mayor el riesgo en el subgrupo de pacientes con disfunción hepática (6). Otros estudios fase II con ixabepilona en monoterapia han demostrado tasa de remisiones completas patológicas en la neoadyuvancia del $18 \%$, y tasa de respuestas de $42 \%$ en primera línea de enfermedad avanzada.

Otro nuevo citostático es la vinflunina. Se trata de un nuevo derivado vincaalcaloide, que ha demostrado su eficacia en el tratamiento de los carcinomas uroteliales avanzados platinorrefractarios, y en segunda línea de carcinoma no microcítico de pulmón. En un estudio fase II de CMI metastático, resistente a taxanos y antraciclinas, el empleo de vinflunina en monoterapia alcanzó una tasa de respuestas del $30 \%$. Actualmente está en marcha un ensayo fase III que compara la eficacia de la combinación de vinflunina con gemcitabina frente a la de paclitaxel con gemcitabina.

Al igual que en otras neoplasias, cada día los nuevos agentes dirigidos frente a dianas terapéuticas adquieren un mayor interés el tratamiento de CMI. De esta forma, podemos añadir a trastuzumab (anticuerpo monoclonal dirigido frente Her-2) (7), otros agentes de reciente aparición como lapatinib, pertuzumab, bevacizumab, y AG 013736, en el tratamiento de CMI.

En el terreno de los agentes dirigidos frente a los receptores transmembrana de factores de crecimiento (familia ErbB), destacan lapatinib y pertuzumab. Estos dos agentes terapéuticos son útiles en el subgrupo de CMI HER-2 positivo. 
- Lapatinib es un inhibidor dual de las tirosin-quinasas del dominio intracelular de los receptores Her-2 y EGFR. Este fármaco ha demostrado su eficacia, en combinación con capecitabina, en CMI metastático (HER-2 positivo) previamente tratado con antraciclinas, taxanos y trastuzumab. En este ensayo fase III, la SLP fue significativamente superior en la rama de la combinación frente a la de capecitabina sola $(8,4 \mathrm{vs} .4,4$ meses, HR =0,49; p <0,001) (8).

-Pertuzumab es un nuevo anticuerpo monoclonal capaz de bloquear la formación de dímeros EGFR/HER-2, esencial para la activación de dichos receptores. Los resultados preliminares obtenidos con este fármaco son prometedores en el tratamiento de CMI HER-2 positivo.

La inhibición de la angiogénesis tumoral ha adquirido una gran importancia en el tratamiento de CMI. Recientemente, se han publicado los resultados definitivos del ensayo clínico fase III, encabezado por Miller K. En este estudio se compara la eficacia de la asociación de bevacizumab con paclitaxel frente a paclitaxel en monoterapia en primeral línea de CMI metastático. Este ensayo con 722 pacientes evidenció un incremento del $40 \%$ en la SLP a favor de la rama experimental (11,8 vs. 5,9 meses; HR $=0,60 ; \mathrm{p}<0,001)$, y también un aumento de la tasa de respuestas $(36,9$ vs. $21,2 \%$; p $<0,001)$. Sin embargo, no hubo un aumento significativo de la supervivencia global $(26,7$ vs. 25,2 meses, $\mathrm{HR}=0,88 ; \mathrm{p}=0,16)(9)$. El estudio AVADO (fase III), que compara la eficacia de dos dosificaciones diferentes de bevacizumab con docetaxel frente a docetaxel en monoterapia en primera línea, va a permitir establecer la dosis más idónea de bevacizumab en CMI. Otros estudios con bevacizumab en enfermedad avanzada, y también en neoadyuvancia y adyuvancia de CMI no metastático están en marcha.

Por último, cabe destacar los resultados obtenidos con AG 013736, presentado en la prestigiosa reunión internacional de ASCO 2007. Se trata de un inhibidor de la actividad tirosinquinasa del receptor del factor de crecimiento del endotelio vascular (VEGFR), y del receptor del factor de crecimiento derivado de plaquetas (PDGFR-beta) y C-KIT. Su combinación con docetaxel en primera línea de CMI avanzado ha conseguido una mejoría en la tasa de respuestas, así como una tendencia a mejorar la SLP.

\section{P. KHOSRAVI SHAHI, G. PÉREZ MANGA}

Servicio de Oncología Médica. Hospital General Universitario Gregorio Marañón. Madrid

\section{Bibliografía}

1. Jemal A, Tiwani RC, Murray T, Ghafoor A, Samuels A,Ward E, et al. Cancer statistics, 2004. CA Cancer J Clin 2004; 54: 8-29.

2. Khosravi P, Izarzugaza Y, Encinas S, et al. Tratamiento adyuvante en el cáncer de mama operable. An Med Interna (Madrid) 2008; 25: 36-40.

3. Khosravi P, Pérez-Manga G. Una nueva estrategia terapéutica en el cáncer de mama: quimioterapia metronómica. An Med Interna (Madrid) 2007; 24: 261-2

4. Khosravi P, Pérez-Manga G. La aplicación de la tecnología de los Microarrays en la Oncología clínica. An Med Interna (Madrid) 2006; 23: 255-6.

5. Khosravi P, Pérez-Manga G. Subtipos clínicos y genéticos de cáncer de mama: individualización del tratamiento. An Med Interna (Madrid)

2007; 24: 569-70.

6. Thomas ES, Gómez HL, Li RK, et al. Ixabepilone plus capecitabine for metastatic breast cancer progressing after anthracycline and taxane treatment. J Clin Oncol 2007; 25: 5210-17.

7. Khosravi P, Pérez-Manga G. La relevancia clínica de la sobreexpresión de HER-2 en el cáncer de mama. An Med Interna (Madrid) 2006; 23: 103-4.

8. Geyer CE, Forster J, Lindquist D, et al. Lapatinib plus capecitabine for HER2-positive advanced breast cancer. N Engl J Med 2006; 355: 2733-43.

9. Miller K, Wang M, Gralow J, et al. Paclitaxel plus Bevacizumab versus Paclitaxel alone for metastatic breast cancer. N Engl J Med 2007; 357 : 2666-76. 